\title{
Touch thresholds as a function of onset and offset stimulation'
}

THOMAS G. STICHT ${ }^{2}$ AND ROBERT H. GIBSON

UNIVERSITY OF PITTSBURGH

Touch thresholds for the application of a nylon thread stimulus to the skin were 6-10 times higher than the thresholds for detecting the subsequent removal of the stimulus. This indicates that, contrary to some other reports, the direction of displacement of the skin may indeed affect the touch threshold.

The removal of a mechanically deforming stimulus from the skin's surface is followed by a "rebound" movement of the skin due to the viscous and elastic properties of the latter. This reformation movement may produce a report of touch if it is of sufficient magnitude (Nafe \& Wagoner, 1941). The purpose of the present study was to determine the threshold for the detection of the removal (offset) of a stimulus from the skin, and to compare this with the threshold for the detection of the application (onset) of the stimulus. Some recent work (Verrillo, 1965) has led to the conclusion that the direction of displacement of the skin has no effect on the threshold for touch. The present results suggest certain restrictions on such a conclusion.

Method

Touch thresholds were determined for three Ss with stimulation of the right thenal produced by means of the Semmes-Weinstein series of calibrated nylon threads (described in detail in Semmes, Weinstein, Ghent, \& Teuber, 1960). When these threads are applied such that only the tips are allowed to touch the skin surface, and a rapid rate of delivery and removal is used, the maximal force exerted is practically instantaneous. A Mettler type H15 analytical balance was used to calibrate the force exerted by the thread. This balance was also used to determine the constancy of the force exerted by each thread chrring the interval between the application and subsequent removal of the thread from the balance. The $<5 \%$ variation in the force during this interval was negligible in view of the large differences subsequently obtained between onset and offset thresholds.

Touch thresholds were obtained using the method of limits with five ascending and five descending trials. In the ascending series the onset threshold was the value of the thread which first produced a sensation of touch when applied to the skin. To obtain the offset threshold, E continued the ascending series until $S$ reported a touch sensation at the removal of the stimulus. In the descending trials, E recorded the value of the stimulus for which $S$ first reported that he could not detect the offset, and then continued descending through the series of threads until S first failed to detect the stimulus onset. This value was taken as the descending threshold. The interval of time between the onset and offset was never less than 5 sec.

\section{Results and Discussion}

The medians of each S's ascending and descending trials were obtained for both onset and offset thresholds. The means of these medians for each $S$ were computed and are presented in Table 1. For all three Ss, the offset thresholds are 6-10 times greater than the onset thresholds. Determinations from two additional observers substantiated these findings.

Considerable evidence (cf., Kenshalo \& Nafe, 1962) has indicated that the tactile receptors respond only to some aspect of the movement of the skin tissues above a minimal rate. This is why no touch response is elicited while the stimulus rests, quiescently, between its application and removal. Observation of the skin following the removal of a slight deforming stimulus reveals that the "rebound" movement is quite slow, when compared to the rapid movement of the tissues produced by the rapid onset of a stimulus. A minimal rate of movement must be exceeded before touch occurs, and the tissue movement produced by the abrupt onset of the stimulus is rapid. Since the reformation movement is slow by contrast, being determined by the tissue properties alone, a relatively large force must be exerted on the skin before it will be placed under sufficient tension to produce a suprathreshold rate of "rebound."13 This being so, it is suggested that the ratio of the offset threshold force to the onset threshold force, reflecting as it does the visco-elastic properties of the tissue, might be used as an index of the resiliency of the skin.

The present results are consistent with earlier studies which have indicated that onset RTs are faster than offset RTs (Sticht, 1965) and that the RT and the threshold for an increase in a pressure

Table 1. Onset and Offset Thresholds in $\mathrm{mg}$.

\begin{tabular}{lcc} 
Subject & Onset & Offset \\
\hline C.L. & 67 & 408 \\
T.S. & 25 & 264 \\
M.M. & 25 & 296 \\
\hline
\end{tabular}


stimulus are lower than for a decrease in the pressure stimulus (Chiba, 1923; Grindley, 1936). It is possible that findings (Verrillo, 1965) of no differences in incremental and decremental pressure thresholds may be due to the use of an initial amplitude of skin deformation of $1 \mathrm{~mm}$, which might produce sufficient tension in the skin tissues to produce rebound movements comparable (with respect to touch thresholds) to those accompanying the compression stimulus. Whatever the case, it appears that the direction of displacement of the skin can, under certain circumstances, affect the threshold for touch.

\section{References}

Chiba, T. Ueber das asymmetrie der Unterschiedsempfindlichkeit. $Z$. Psychol., 1923, 92, 177-226.

Grindley, G. C. The variation of sensory thresholds with the rate of application of the stimulus: I. The differential threshold for pressure. Brit. J. Psychol., 1936, 27, 86-95.

Kenshalo, D. R., \& Nafe, J. P. A quantitative theory of feeling. Psychol. Rev., 1962, 69, 17-33.

Nafe, J. P., \& Wagoner, K. S. The nature of sensory adaptation. J. gen. Psychol., 1941, 25, 295-322.
Semmes, J., Weinstein, S., Ghent, L., \& Teuber, H. L. Somatosensory changes after penetrating brain wounds in man. Cambridge: Harvard University Press, 1960.

Sticht, T. G. Reaction time to cutaneous onset and offset stimulation. Percept. mot. Skills, 1964, 19, 611-614.

Sticht, T. G., \& Sitterley, T. E. Frequency and latency of response to offset-onset sequences of cutaneous stimulation. Psychon. Sci., 1965, 3, 425-426.

Verrillo, R. T. Vibrotactile threshold and pulse polarity. Psychon. Sci., 1965, 3, 171 .

\section{Notes}

1. This research was supported in part by the following grants to Robert H. Gibson: National Science Foundation, grant No. GB3268, National Institutes of Neurological Diseases and Blindness, grant No. NB-06304, and Health Research and Services Foundation, grant No. I-11.

2. Space Research and Coordination Center Postdoctoral Fellow, Department of Psychology, 1966-67.

3. By increasing the time interval between the removal of a probe and its subsequent replacement onto the skin, the extent of the reformation movement which results from visco-elastic properties of the tissue, is increased. Using such stimuli, threshold detectability of touch increases as the off duration increases (Sticht \& Sitterley, 1965). 